\title{
On the caustics of Bézier curves
}

\author{
Imre Juhász \\ Department of Descriptive Geometry, University of Miskolc \\ imre.juhasz@uni-miskolc.hu \\ Submitted: June 6, 2019 \\ Accepted: November 22, 2019 \\ Published online: November 22, 2019
}

\begin{abstract}
We provide exact formulae for the rational Bézier representation of caustics of planar Bézier curves of degree greater than one.
\end{abstract}

Keywords: caustic, Bézier curve

$M S C: 65 \mathrm{D} 17,68 \mathrm{U} 07$

\section{Introduction}

In optics a caustic is the envelope of light rays reflected or refracted by an object. We consider only that special case when the rays are parallel and are reflected by a planar curve.

Recently, caustics of control point based planar curves were studied in [6], however the special properties of basis functions in use were not exploited. In the present contribution we concentrate on the caustics of planar Bézier curves, the basis functions of which are the Bernstein polynomials.

\section{Caustic curve}

Without the loss of generality we can assume that the direction of the light rays is $\left[\begin{array}{ll}1 & 0\end{array}\right]^{T}$, since this only results in an isometric transformation of the curve. We consider the sufficiently smooth curve

$$
\mathbf{r}(t)=\left[\begin{array}{l}
r_{x}(t) \\
r_{y}(t)
\end{array}\right], t \in[a, b]
$$


The direction of the reflected ray at the point $\mathbf{r}(t)$ is

$$
\mathbf{v}(t)=\left[\begin{array}{r}
1-\frac{2 \dot{r}_{y}^{2}(t)}{\dot{r}_{x}^{2}(t)+\dot{r}_{y}^{2}(t)} \\
\frac{2 \dot{r}_{x}(t) \dot{r}_{y}(t)}{\dot{r}_{x}^{2}(t)+\dot{r}_{y}^{2}(t)}
\end{array}\right], t \in[a, b]
$$

and the caustic $\mathbf{c}$ of the curve $\mathbf{r}$ can be written in the form

$$
\begin{aligned}
& c_{x}(t)=r_{x}(t)+\frac{\left(\dot{r}_{x}^{2}(t)-\dot{r}_{y}^{2}(t)\right) \dot{r}_{y}(t)}{2\left(\dot{r}_{x}(t) \ddot{r}_{y}(t)-\ddot{r}_{x}(t) \dot{r}_{y}(t)\right)}, \\
& c_{y}(t)=r_{y}(t)+\frac{\dot{r}_{x}(t) \dot{r}_{y}^{2}(t)}{\dot{r}_{x}(t) \ddot{r}_{y}(t)-\ddot{r}_{x}(t) \dot{r}_{y}(t)} .
\end{aligned}
$$

An equivalent of the above formula for the caustic was also derived in [6].

The caustic may have point(s) at infinity, i.e., the curve can be composed of several branches. This happens where the denominator $\dot{r}_{x}(t) \ddot{r}_{y}(t)-\ddot{r}_{x}(t) \dot{r}_{y}(t)$ vanishes, i.e., where the curvature of $\mathbf{r}$ is zero. The asymptote at such a point is the reflected ray $\mathbf{r}(t)+\lambda \mathbf{v}(t), \lambda \in \mathbb{R}$ itself. In Fig. 1 there is a quartic Bézier curve the caustic of which has two points at infinity.

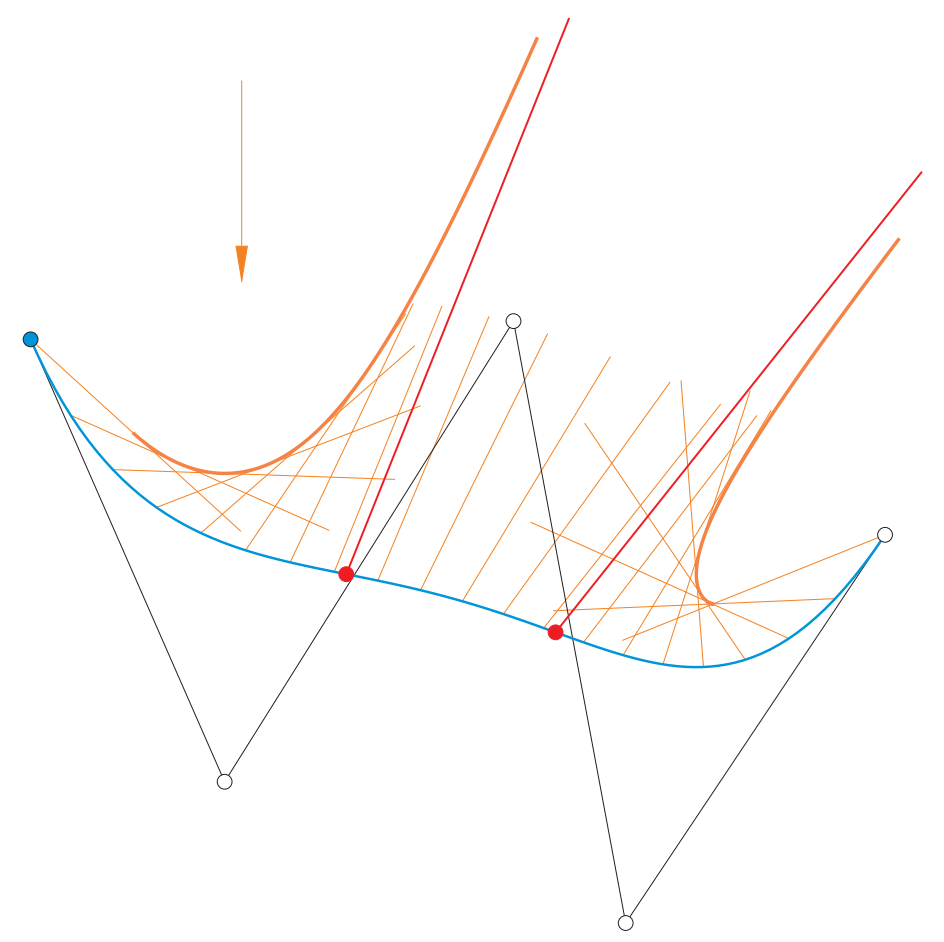

Figure 1: A quartic Bézier curve along with its caustic, which has two points at infinity. The arrow indicates the light direction. 
From here on, we will study the caustics of planar Bézier curves

$$
\mathbf{r}(t)=\sum_{i=0}^{n} B_{i}^{n}(t) \mathbf{b}_{i}, t \in[0,1], n \geq 2
$$

where the sequence of points $\left\{\mathbf{b}_{i}\right\}_{i=0}^{n}$ are called control points and $B_{i}^{n}$ denotes the $i$ th Bernstein polynomial of degree $n$. (The case $n=1$ is out of interest, since then the curve degenerates to a straight line segment and the reflected rays are parallel.)

\section{Caustic of a Bézier curve}

At first, we reformulate the caustic $\mathbf{c}$ to have a common denominator of the the coordinate functions, yielding

$$
\begin{aligned}
c_{x}(t) & =\frac{2\left(\dot{r}_{x}(t) \ddot{r}_{y}(t)-\ddot{r}_{x}(t) \dot{r}_{y}(t)\right) r_{x}(t)+\dot{r}_{x}^{2}(t) \dot{r}_{y}(t)-\dot{r}_{y}^{3}(t)}{2\left(\dot{r}_{x}(t) \ddot{r}_{y}(t)-\ddot{r}_{x}(t) \dot{r}_{y}(t)\right)}, \\
c_{y}(t) & =\frac{2\left(\dot{r}_{x}(t) \ddot{r}_{y}(t)-\ddot{r}_{x}(t) \dot{r}_{y}(t)\right) r_{y}(t)+2 \dot{r}_{x}(t) \dot{r}_{y}^{2}(t)}{2\left(\dot{r}_{x}(t) \ddot{r}_{y}(t)-\ddot{r}_{x}(t) \dot{r}_{y}(t)\right)} .
\end{aligned}
$$

Obviously, numerators of the above expressions are polynomials of degree $3(n-1)$ and the common denominator is of degree $(2 n-3)$, therefore these coordinate functions are rational functions of degree $3(n-1)$. In what follows we provide the rational Bézier representation of such caustics. We introduce notations

$$
\begin{aligned}
\dot{\mathbf{r}}(t) & =\sum_{i=0}^{n-1} B_{i}^{n-1}(t) \mathbf{a}_{i}, t \in[0,1], \mathbf{a}_{i}=n\left(\mathbf{b}_{i+1}-\mathbf{b}_{i}\right), i=0,1, \ldots, n-1, \\
\ddot{\mathbf{r}}(t) & =\sum_{i=0}^{n-2} B_{i}^{n-2}(t) \mathbf{d}_{i}, t \in[0,1], \mathbf{d}_{i}=(n-1)\left(\mathbf{a}_{i+1}-\mathbf{a}_{i}\right), i=0,1, \ldots, n-2 .
\end{aligned}
$$

Making use of the identity

$$
B_{i}^{n}(t) B_{j}^{m}(t)=\frac{\left(\begin{array}{c}
n \\
i
\end{array}\right)\left(\begin{array}{c}
m \\
j
\end{array}\right)}{\left(\begin{array}{c}
n+m \\
i+j
\end{array}\right)} B_{i+j}^{n+m}(t)
$$

of Bernstein polynomials (cf. [1]), we can derive an identity for the product of two linear combinations $\sum_{i=0}^{n} B_{i}^{n}(t) a_{i}$ and $\sum_{j=0}^{m} B_{j}^{m}(t) b_{j}$ can be written in the form

$$
\sum_{i=0}^{n} B_{i}^{n}(t) a_{i} \sum_{j=0}^{m} B_{j}^{m}(t) b_{j}=\sum_{\ell=0}^{n+m} B_{\ell}^{n+m}(t) \frac{1}{\left(\begin{array}{c}
n+m \\
\ell
\end{array}\right)} \sum_{k=0}^{m}\left(\begin{array}{c}
n \\
\ell-k
\end{array}\right)\left(\begin{array}{c}
m \\
k
\end{array}\right) a_{\ell-k} b_{k}
$$

provided $n \geq m$. 
Now, we study the common denominator. By means of identity 3.3, its first term is of the form

$$
\begin{aligned}
\dot{r}_{x}(t) \ddot{r}_{y}(t) & =\sum_{i=0}^{n-1} B_{i}^{n-1}(t) a_{x, i} \sum_{j=0}^{n-2} B_{j}^{n-2}(t) d_{y, j} \\
& =\sum_{\ell=0}^{2 n-3} B_{\ell}^{2 n-3}(t) \frac{1}{\left(\begin{array}{c}
2 n-3 \\
\ell
\end{array}\right)} \sum_{k=0}^{n-2}\left(\begin{array}{c}
n-1 \\
\ell-k
\end{array}\right)\left(\begin{array}{c}
n-2 \\
k
\end{array}\right) a_{x, \ell-k} d_{y, k} .
\end{aligned}
$$

The second term can analogously be expressed, yielding

$$
\dot{r}_{y}(t) \ddot{r}_{x}(t)=\sum_{\ell=0}^{2 n-3} B_{\ell}^{2 n-3}(t) \frac{1}{\left(\begin{array}{c}
2 n-3 \\
\ell
\end{array}\right)} \sum_{k=0}^{n-2}\left(\begin{array}{c}
n-1 \\
\ell-k
\end{array}\right)\left(\begin{array}{c}
n-2 \\
k
\end{array}\right) a_{y, \ell-k} d_{x, k}
$$

and the denominator has the form

$$
2\left(\dot{r}_{x}(t) \ddot{r}_{y}(t)-\ddot{r}_{x}(t) \dot{r}_{y}(t)\right)=\sum_{\ell=0}^{2 n-3} w_{\ell} B_{\ell}^{2 n-3}(t)
$$

where

$$
w_{\ell}=\frac{2}{\left(\begin{array}{c}
2 n-3 \\
\ell
\end{array}\right)} \sum_{k=0}^{n-2}\left(\begin{array}{c}
n-1 \\
\ell-k
\end{array}\right)\left(\begin{array}{c}
n-2 \\
k
\end{array}\right)\left(a_{x, \ell-k} d_{y, k}-a_{y, \ell-k} d_{x, k}\right) .
$$

We elevate the degree of (3.4) by $n$, using the general degree elevation formula

$$
\begin{aligned}
\sum_{i=0}^{s} B_{i}^{s}(t) w_{i} & =\sum_{i=0}^{s+z} B_{i}^{s+z}(t) w_{i}^{[z]}, z>0, \\
w_{i}^{[z]} & =w_{i}^{[z-1]}+\frac{i}{s+z}\left(w_{i-1}^{[z-1]}-w_{i}^{[z-1]}\right), i=0,1, \ldots, s+z \\
w_{i}^{[0]} & =w_{i}, i=0,1, \ldots, s .
\end{aligned}
$$

with substitutions $s=3(n-1)$ and $z=n$.

The degree elevated denominator is

$$
\sum_{\ell=0}^{3(n-1)} w_{\ell}^{[n]} B_{\ell}^{3(n-1)}(t), t \in[0,1]
$$

The numerator of the $x$ coordinate function is

$$
2\left(\dot{r}_{x}(t) \ddot{r}_{y}(t)-\ddot{r}_{x}(t) \dot{r}_{y}(t)\right) r_{x}(t)+\dot{r}_{x}^{2}(t) \dot{r}_{y}(t)-\dot{r}_{y}^{3}(t) .
$$

Its first term can be expressed as

$$
2\left(\dot{r}_{x}(t) \ddot{r}_{y}(t)-\ddot{r}_{x}(t) \dot{r}_{y}(t)\right) r_{x}(t)
$$




$$
=\sum_{\ell=0}^{3(n-1)} B_{\ell}^{3(n-1)}(t) \frac{1}{\left(\begin{array}{c}
3(n-1) \\
\ell
\end{array}\right)}\left(\sum_{k=0}^{n}\left(\begin{array}{c}
2 n-3 \\
\ell-k
\end{array}\right)\left(\begin{array}{l}
n \\
k
\end{array}\right) w_{\ell-k} b_{x, k}\right)
$$

and the second one as

$$
\begin{aligned}
\dot{r}_{x}^{2}(t) \dot{r}_{y}(t)= & \sum_{\ell=0}^{3(n-1)} B_{\ell}^{3(n-1)}(t) \frac{1}{\left(\begin{array}{c}
3(n-1) \\
\ell
\end{array}\right)} \\
& \times \sum_{k=0}^{n-1}\left(\left(\begin{array}{c}
n-1 \\
k
\end{array}\right) a_{y, k} \sum_{z=0}^{n-1}\left(\begin{array}{c}
n-1 \\
\ell-k-z
\end{array}\right)\left(\begin{array}{c}
n-1 \\
z
\end{array}\right) a_{x, \ell-k-z} a_{x, z}\right)
\end{aligned}
$$

while the third one as

$$
\begin{aligned}
\dot{r}_{y}^{3}(t)= & \sum_{\ell=0}^{3(n-1)} B_{\ell}^{3(n-1)}(t) \frac{1}{\left(\begin{array}{c}
3(n-1) \\
\ell
\end{array}\right)} \\
& \times \sum_{k=0}^{n-1}\left(\left(\begin{array}{c}
n-1 \\
k
\end{array}\right) a_{y, k} \sum_{z=0}^{n-1}\left(\begin{array}{c}
n-1 \\
\ell-k-z
\end{array}\right)\left(\begin{array}{c}
n-1 \\
z
\end{array}\right) a_{y, \ell-k-z} a_{y, z}\right) .
\end{aligned}
$$

Thus, the numerator of Eq. (3.1) can be written in the form

$$
\sum_{\ell=0}^{3(n-1)} B_{\ell}^{3(n-1)}(t) q_{x, \ell},
$$

where

$$
\begin{aligned}
q_{x, \ell}= & \frac{1}{\left(\begin{array}{c}
3(n-1) \\
\ell
\end{array}\right)}\left(\sum_{k=0}^{n}\left(\begin{array}{c}
2 n-3 \\
\ell-k
\end{array}\right)\left(\begin{array}{l}
n \\
k
\end{array}\right) w_{\ell-k} b_{x, k}+\sum_{k=0}^{n-1}\left(\begin{array}{c}
n-1 \\
k
\end{array}\right) a_{y, k}\right. \\
& \left.\times \sum_{z=0}^{n-1}\left(\begin{array}{c}
n-1 \\
\ell-k-z
\end{array}\right)\left(\begin{array}{c}
n-1 \\
z
\end{array}\right)\left(a_{x, \ell-k-z} a_{x, z}-a_{y, \ell-k-z} a_{y, z}\right)\right) .
\end{aligned}
$$

Analogously, we can obtain the numerator of (3.2) in the form

$$
\sum_{\ell=0}^{3(n-1)} B_{\ell}^{3(n-1)}(t) q_{y, \ell}
$$

where

$$
\begin{aligned}
q_{y, \ell}= & \frac{1}{\left(\begin{array}{c}
3(n-1) \\
\ell
\end{array}\right)}\left(\sum_{k=0}^{n}\left(\begin{array}{c}
2 n-3 \\
\ell-k
\end{array}\right)\left(\begin{array}{l}
n \\
k
\end{array}\right) w_{\ell-k} b_{y, k}+2 \sum_{k=0}^{n-1}\left(\begin{array}{c}
n-1 \\
k
\end{array}\right) a_{x, k}\right. \\
& \left.\times \sum_{z=0}^{n-1}\left(\begin{array}{c}
n-1 \\
\ell-k-z
\end{array}\right)\left(\begin{array}{c}
n-1 \\
z
\end{array}\right) a_{y, \ell-k-z} a_{y, z}\right) .
\end{aligned}
$$


Finally, the rational Bézier representation of the caustic curve is

$$
\mathbf{c}(t)=\sum_{\ell=0}^{3(n-1)} \frac{w_{\ell}^{[n]} B_{\ell}^{3(n-1)}(t)}{\sum_{k=0}^{3(n-1)} w_{k}^{[n]} B_{k}^{3(n-1)}(t)} \mathbf{q}_{\ell}, t \in[0,1]
$$

where weights $\left\{w_{\ell}^{[n]}\right\}_{\ell=0}^{3(n-1)}$ are the coefficients obtained by the degree elevation of the denominator, and control points are specified by

$$
\mathbf{q}_{\ell}=\frac{1}{w_{\ell}^{[n]}}\left[\begin{array}{l}
q_{x, \ell} \\
q_{y, \ell}
\end{array}\right], \ell=0,1, \ldots, 3(n-1) .
$$

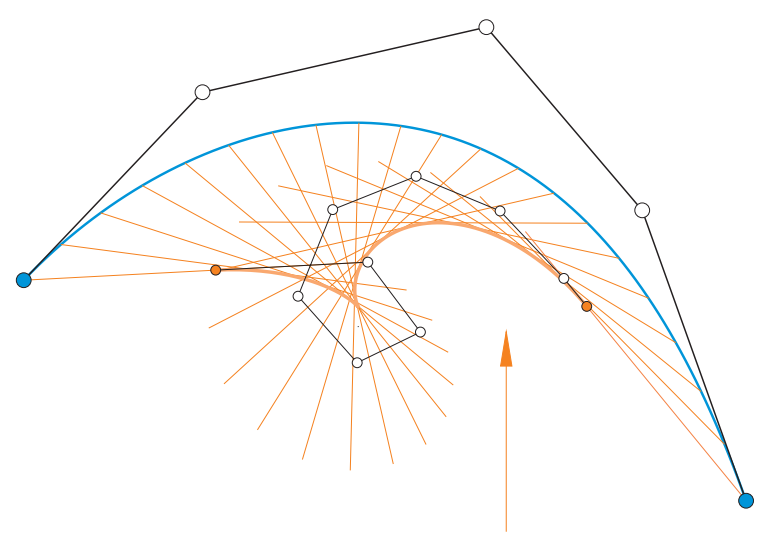

Figure 2: A quartic Bézier curve and its caustic along with the reflected rays. The control polygon of the rational Bézier representation (which is of degree 9) is also displayed. The arrow indicates the light direction.

Now, we summarize our results.

Proposition 3.1. The caustic of a Bézier curve of degree $n$ (if exists) is a rational Bézier curve of degree $3(n-1)$. Its weights and control points are specified by $(3.5)$, (3.6) and (3.7), (3.8), (3.9), respectively.

Remark 3.2. The caustic of a quadratic Bézier curve $(n=2)$ (if exists) is a cubic polynomial curve, since in this case the common denominator of (3.1) and (3.2) is the constant

$$
\begin{aligned}
& \sum_{\ell=0}^{1} B_{\ell}^{1}(t)\left(2 \sum_{k=0}^{1} \frac{\left(\begin{array}{l}
2-1 \\
\ell-k
\end{array}\right)\left(\begin{array}{l}
0 \\
k
\end{array}\right)}{\left(\begin{array}{l}
1 \\
\ell
\end{array}\right)}\left(a_{x, \ell-k} d_{y, k}-a_{y, \ell-k} d_{x, k}\right)\right) \\
& =2 \sum_{\ell=0}^{1} B_{\ell}^{1}(t)\left(a_{x, \ell} d_{y, 0}-a_{y, \ell} d_{x, 0}\right)
\end{aligned}
$$




$$
\begin{aligned}
& =2\left(a_{x, 0} a_{y, 1}-a_{x, 1} a_{y, 0}\right)\left(B_{0}^{1}(t)+B_{1}^{1}(t)\right) \\
& =2\left(a_{x, 0} a_{y, 1}-a_{x, 1} a_{y, 0}\right),
\end{aligned}
$$

therefore the caustic is a cubic polynomial curve. It is well-known that the caustic of a parabola is a Tschirnhausen cubic, if the rays are not parallel to the axis of the parabola, we have just obtained its Bézier representation.

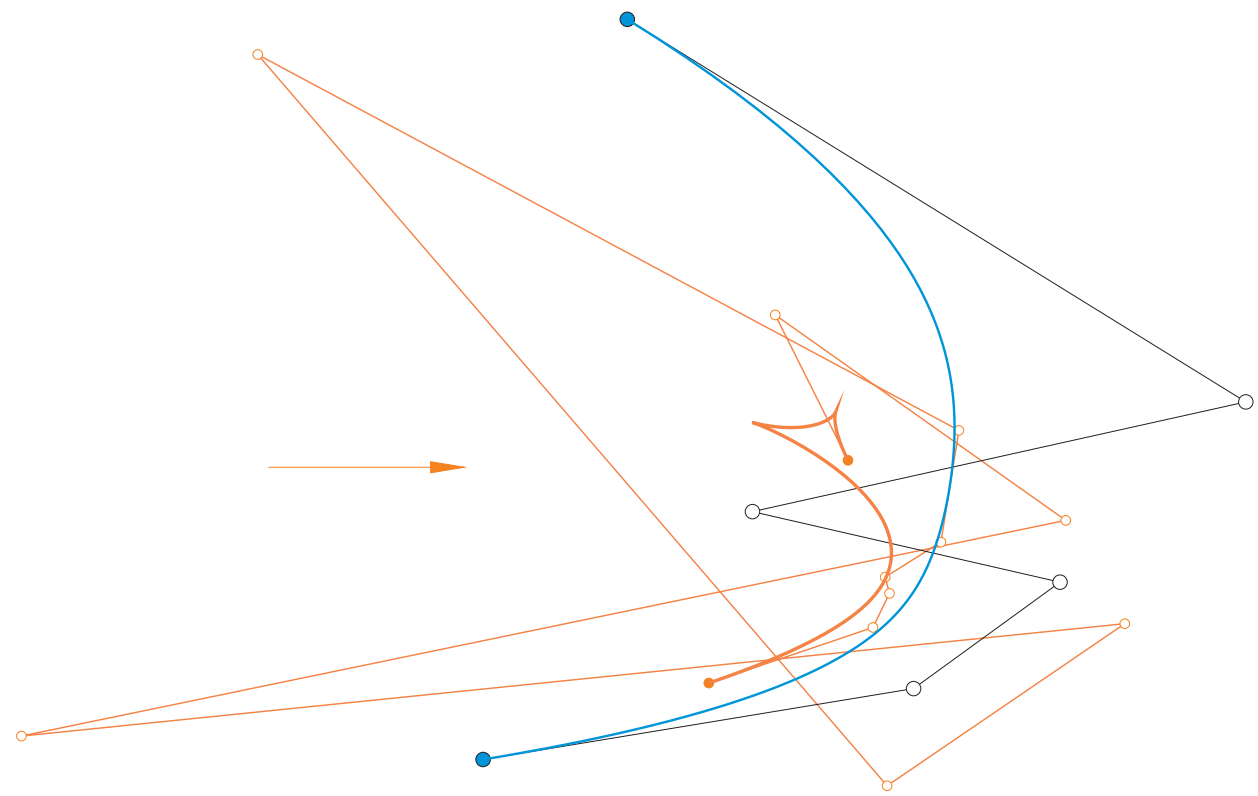

Figure 3: A quintic Bézier curve the caustic of which has two cusps. The control polygon of the rational Bézier representation of the caustic is also shown. The arrow indicates the light direction.

Remark 3.3. The cusp(s) of the caustic may be of interest. The caustic $\mathbf{c}$ has a cusp at $t_{0} \in[0,1]$, if $\left\|\dot{\mathbf{c}}\left(t_{0}\right)\right\|=0$, i.e., if the tangent vector vanishes, that we can find numerically. Actually, it is a root finding problem, which can be solved efficiently with high precision and stability, since the polynomials are specified in Bernstein basis (cf. $[2,5]$ ).

In Fig. 2 there is a quartic Bézier curve and its caustic, along with the control polygon of the rational Bézier representation of the caustic. Fig. 3 shows such a quintic Bézier curve whose caustic has two cusps.

\section{Conclusions}

We have provided ready to implement exact formulae for the rational Bézier representation of caustics (if exist) of planar Bézier curves of degree greater than 
one. This method can be extended to other control point based curves, i.e., curves described in the form

$$
\mathbf{c}(t)=\sum_{i=0}^{n} F_{i}^{n}(t) \mathbf{d}_{i}, t \in[a, b] .
$$

We assume that function system $\mathcal{F}:=\left\{F_{i} \mid F_{i}:[a, b] \rightarrow \mathbb{R}\right\}_{i=0}^{n}$ consists of sufficiently smooth non-negative functions, forming a partition of unity. Additional requirements are the existence of degree elevation and product formulae in the basis $\mathcal{F}$. These requirements are fulfilled by the B-basis of trigonometric (cf. [3]) and that of hyperbolic polynomials (cf. [4]), besides the Bernstein basis.

\section{References}

[1] G. FARIN: Curves and surfaces for CAGD: a practical guide, 5th edition, Morgan Kaufmann, 2001, DOI: $10.1016 /$ B978-1-55860-737-8.X5000-5.

[2] R. T. Farouki, V. Rajan: Algorithms for polynomials in Bernstein form, Computer Aided Geometric Design 5.1 (1988), pp. 1-26, DOI: 10.1016/0167-8396(88)90016-7.

[3] J. SÁnchez-Reyes: Harmonic rational Bézier curves, p-Bézier curves and trigonometric polynomials, Computer Aided Geometric Design 15.9 (1998), pp. 909-923, DoI: 10.1016 / S0167-8396 (98) 00031-4.

[4] W.-Q. Shen, G.-Z. Wang: A class of quasi Bézier curves based on hyperbolic polynomials, Journal of Zhejiang University Science 6.1 (2005), pp. 116-123, DOI: 10.1007/BF02887226.

[5] M. R. Spencer: Polynomial real root finding in Bernstein form, PhD thesis, Brigham Young University, 1994, URL: https://scholarsarchive.byu.edu/etd/4246.

[6] E. Troll, M. Hoffmann: Caustics of spline curves, Annales Mathematicae et Informaticae 47 (2017), pp. 201-209, URL: http : / /ami . ektf . hu/uploads/papers/finalpdf / AMI\% 5Ctextunderscore $47 \% 5$ Ctextunderscore\% 20 from 201 to 209 .pdf. 